\title{
Moringa fatty acid methyl esters as a source of platform chemicals developed via meathesis route
}

\author{
Vyshnavi Yelchuri, R.B.N. Prasad, M.S.L. Karuna* \\ Centre for Lipid Research, CSIR-Indian Institute of Chemical Technology, Hyderabad 500007, India
}

\begin{abstract}
Self-metathesis of fatty acids is an attractive modern method to synthesize platform chemicals useful for a wide variety of industrial applications. Degraded moringa seeds rich in mono unsaturation (75\%), which are no longer useful for edible purpose have been utilized for the development of platform chemicals employing self-metathesis using Grubbs' second generation catalyst. Seeds were soxhlet extracted and the fatty acid methyl esters were subjected to self-metathesis using Grubbs' second generation catalyst with $0.3 \mathrm{mM}$ concentration at $40-45{ }^{\circ} \mathrm{C}$ for $36 \mathrm{~h}$, which resulted in products namely, 9-Octadecene (15\%), 6-pentadecene (9.3\%), 3-dodecene (6.7\%), Cyclodecacycltetradecene (14\%), 8,11- Eicosadienoic acid methyl ester (9.7\%) and Cyclopropaneoctanoic acid methyl ester (5.1\%). 9- Octadecene, a lube range hydrocarbon and other platform chemicals with potential as synthetic intermediates have been developed. Hence, the discarded moringa seeds can form a potential alternative raw material for the generation of organic intermediates.
\end{abstract}

Keywords: Moringa fatty acid methyl esters, Grubbs' catalyst, platform chemicals, 9-octadecene.

\section{Introduction}

Designing of new processes are today focused more towards nontoxic materials, save energy and generate less waste. New catalytic methods (chemical catalysis and biocatalysis) are being developed over the last few years with a focus to reduce number of steps, "atom economy" and energy reduction to produce renewable raw materials with high chemo-, regio- and stereo-selectivity. Vegetable or plant oils with unsaturated fatty acids form promising, renewable and cheaper feed stocks for generating a number of oleochemicals for a sustainable future [1-4]. Numbers of pure fatty acids are today available which make them attractive for the synthesis and as raw materials for the chemical industry [3]. The intermediates required for generating a variety of oleochemicals are today fossil-based and these can be generated by conducting a number of organic reactions across the double bonds of vegetable oil fatty acids like oxidations, reductions, polymerizations [5-6] to prepare a wide range of organic intermediates. In this context, olefin metathesis is one of the important and reliable methods for the development of raw materials with strong environmental impact, eco-design with predictive methods into new processes.

Self-metathesis of long chain unsaturated fatty acid esters in the presence of a suitable catalyst provide a convenient and highly selective route to produce unsaturated diesters, high value intermediates for a number of oleochemicals. The most studied fatty acid is methyl oleate (methyl cis-9-octadecenoate), a readily available ester of naturally occurring, cheap oleic acid which yields $50 \%$ (equilibrium conversion) of 9-octadecene (a lube oil range hydrocarbon intermediate) and dimethyl 9-octadecenoate used for the production of macro cyclic compounds on self-metathesis. The diester of methyl or ethyl oleate on subjecting to Dieckmann Condensation leads to civetone, an important base material in perfumery industry [7-8]. These type of products can be prepared in a single step by self metathesis of fatty acids employing cheaper catalysts. Self- metathesis of many long chain unsaturated fatty acids such as methyl elaidate, methyl 11-eicosenoate, methyl palmitoleate, methyl vaccinate, methyl erucate (major fatty acid of rapeseed oil and crambe oil), methyl petroselenate (major fatty acid of coriander oil) and methyl 5-eicosenoate (methyl gadoleate, major fatty acid of meadow foam oil), methyl ricinoleate (major fatty acid of castor oil), methyl undecenoate (second generation product of castor oil) and long chain fatty acids such as methyl linoleate and methyl linolenate were also studied. The various intermediates produced from these sources were polyenes, monoesters, diesters and cyclopolyenes [9-12]. Nonedible oils rich in unsaturation such as rubber ( $80.7 \%$ unsatursation), palash (65.1\% unsaturation) and tobacco (88.2\% unsatiration) were subjected to self metathesis which resulted in a range of novel molecules such as cyclodecacyclododecene, 6-pentadecene, 3-dodecene, 9-octadecenoicacid methyl ester, cyclopropaneoctanoic acid methyl ester along with diesters and 9-octadecene for the first time.

India is one of the largest importers of vegetable oils in the world in the past few years and it is expected to reach 8.5 MMT by the year 2020. In this context, Moringa grown extentively in India for edible applications are easily prone to decomposition on storage for a longer period which are thrown into waste. The seeds contain an oil content of $42 \%$ and also rich in oleic acid content $74.08 \%$, which has the potential to be utilized as an alternate source of oil for developing platform chemicals. Moringa oleifera belongs to the Moringaceae family, a few known varieties namely Jaffna, Chauakacheri Murunga, Chem, Kadu, Palmurungai 
and Periyakulam 1 (PKM 1) [13]. Moringa oleifera commonly called Drumstick or Horse Radish Tree (Hindi Sainjna), is a fast growing tree. Seedlings are grown upto $4 \mathrm{~m}$ in height within 12 months, flowers and fruits are observed within 6 months after planting. The seedpods are left to mature and dry naturally on the tree before harvesting. The seeds are easily shelled, crushed and sieved using traditional methods. The seed oil is known as "Ben oil", or "Behen oil" is rich in oleic acid (omega -9- fatty acid), has strong oxidation stability, longer storage which is suitable for edible purpose, due to the presence of tocopherols, phenolic compounds, steroids and carotenoids and natural antioxidants. Globally drumsticks are used for a wide range of food applications. It is used in lubricant, cosmetic (skin lotions and perfumes), biodiesel, medicinal, antifungal, antimicrobial applications and also utilized for waste water treatment [14-15]. In the present study, the seed oil was subjected to self-metathesis to develop some industrial platform chemicals as the oil is rich in unsaturation.

\subsection{Materials}

\section{Materials and Methods}

The Moringa dry seeds from mature fruits were harvested from drought (Layyah) and irrigated [Jhang (Chenab Nagar), Rahym Yar Khan (Sadiqabad)] regions of the Punjab province of Pakistan. Tricyclohexylphosphine - [1,3-bis(2,4,6-trimethylphenyl) - 4,5 - dihydroimidazol-2-ylidene]benzylidene ruthenium (IV) dichloride (Grubbs second generation catalyst) (II), sulfuric acid, dry methanol, dry dichloromethane (99.9\%) were purchased from M/s Sigma Aldrich.

\subsection{Methods}

2.2.1 Oil extraction: Dried M. oleifera seeds (100 g) were ground, placed into a cellulose paper cone and extracted using light hexane in a $5 \mathrm{~L}$ Soxhlet extractor for $8 \mathrm{~h}$. The oil was then recovered by evaporating the solvent using rotary evaporator and residual solvent was removed by drying in an under high vacuum at $60{ }^{\circ} \mathrm{C}$ for $1 \mathrm{~h}$ and flushing with $99.9 \%$ nitrogen. (42.79 $\mathrm{g}, 42 \%$ yield).

\subsubsection{Gas Chromatography analysis:}

After each extraction, the fatty acid composition of moringa fatty acid methyl esters and the products obtained after self-metathesis were analyzed using an Agilent 6890 gas chromatograph (Agilent Technologies, Palo Alto, CA, USA) fitted with an FID detector and split/splitless injector. A non-bonded cyano silicone column (DB-225, $30 \mathrm{~m}$ x0.32 i.d., J\&W Scientific, USA) was employed for gas chromatography analysis. The column temperature was initially maintained at $100{ }^{\circ} \mathrm{C}$ for $2 \mathrm{~min}$, increased to $300{ }^{\circ} \mathrm{C}$ for a hold time of $20 \mathrm{~min}$ with a flow rate of $10{ }^{\circ} \mathrm{C} / \mathrm{min}$. The injector and detector temperatures were set at $250{ }^{\circ} \mathrm{C}$. Chemstation software was used for the data analysis.

\subsubsection{GC-MS analysis of moringa metathesized products}

The structure of the products obtained by self-metathesis of moringa methyl ester was confirmed using an Agilent 6890N (Agilent Technologoes, Palo Alto, CA, USA) Series equipped with an DB-225 Column (30 m x $0.25 \mathrm{~mm}$ i.d) connected to an Agilent 5973, Mass Spectrometer operating in the EI mode (70 eV; m.z 50550 ; source temperature $230{ }^{\circ} \mathrm{C}$ and a quadruple temperature $150{ }^{\circ} \mathrm{C}$ ). The column temperature was initially maintained at $100{ }^{\circ} \mathrm{C}$ for $2 \mathrm{~min}$, increased to $300{ }^{\circ} \mathrm{C}$ at $10{ }^{\circ} \mathrm{C} / \mathrm{min}$ with a hold time of $20 \mathrm{~min}$ at $300{ }^{\circ} \mathrm{C}$. The inlet temperature was maintained at $300{ }^{\circ} \mathrm{C}$ and split ratio of 50:1. Structural assignments were based on interpretation of mass spectrometric fragmentation and confirmed by comparison of retention times as well as fragmentation pattern of the authentic compounds and the spectral data obtained from the Wiley and NIST libraries.

\subsubsection{Self-metathesis of moringa fatty acid methyl ester}

Moringa fatty acid methyl esters $(2 \mathrm{~g}, 6.1 \mathrm{mM})$ dissolved in dry DCM $20 \mathrm{ml}$ was taken into a two necked round bottom flask under $\mathrm{N}_{2}$ atmosphere. Grubb's second generation catalyst (17 $\left.\mathrm{mg}, 0.3 \mathrm{mM}\right)$ taken in dry DCM was added to the methyl esters using a $20 \mathrm{ml}$ syringe. The contents were heated at $40-45{ }^{\circ} \mathrm{C}$ for 36 $\mathrm{h}$. The reaction was monitored hourly using TLC eluted with hexane: ethyl acetate, 90:10 (v/v). The contents were washed with ethyl acetate and passed over anhydrous sodium sulphate. The solvent was removed using rota evoparator and dried under reduced pressure $(2-5 \mathrm{~mm} \mathrm{Hg})$ to obtain metathesized products $(1.41 \mathrm{~g}, 70 \%$ yield).The products were qualitatively and quantitatively identified using GC and GC-MS.

Table 1. Fatty acids composition (wt \%) of moringa fatty acid methyl esters

\begin{tabular}{|l|l|l|l|l|l|l|l|l|}
\hline Oil & & \multicolumn{6}{|c|}{ Fatty acid composition (wt\%) } \\
\hline & $16: 0$ & $16: 1$ & $18: 0$ & $18: 1$ & $18: 2$ & $20: 0$ & $20: 1$ & $22: 0$ \\
\hline Moringa & 7.03 & 1.54 & 6.17 & 74.08 & 1.22 & 3.4 & 1.24 & 4.01 \\
\hline
\end{tabular}




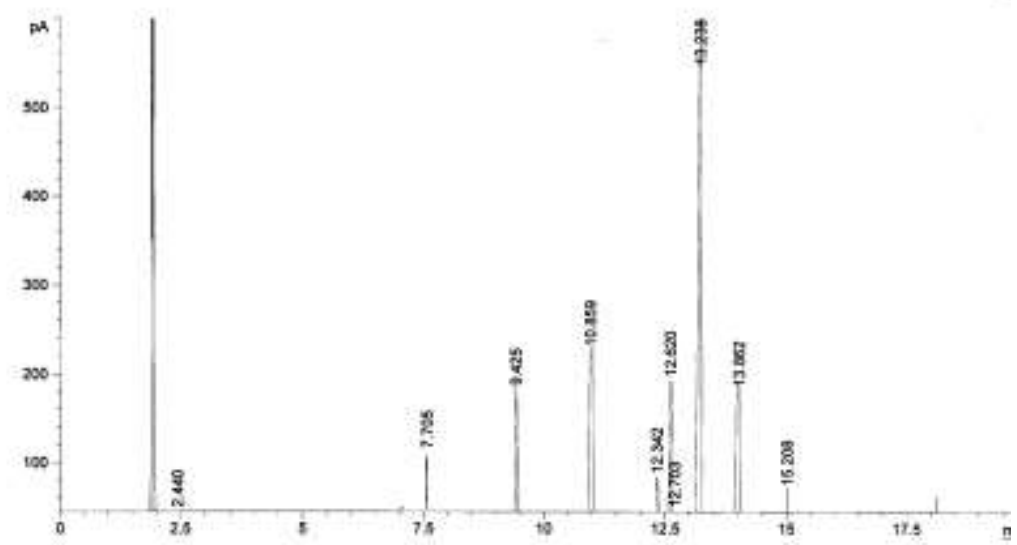

Figure 1. GC of Moringa fatty acid methyl esters

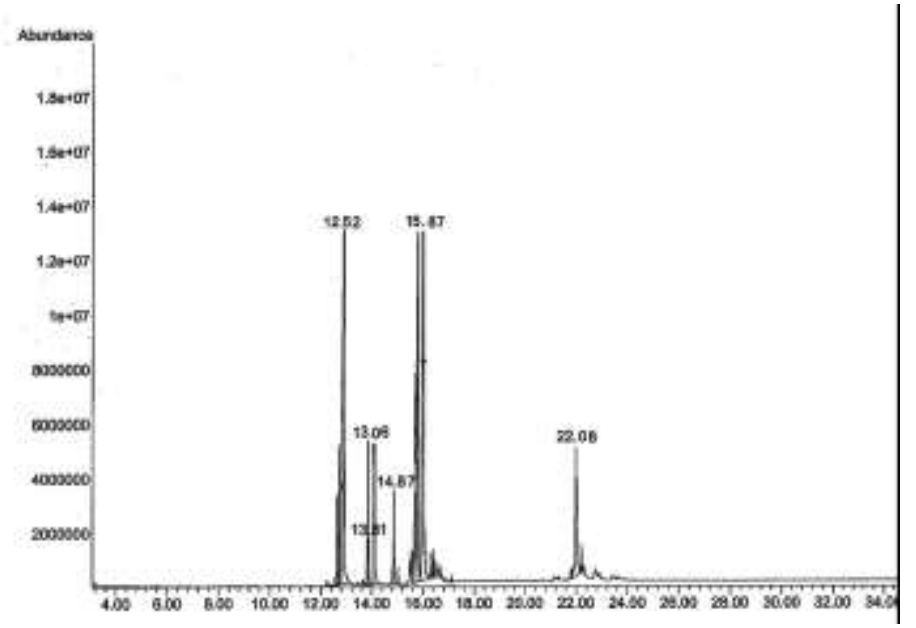

Figure 2. GC of self-metathesis of moringa fatty acid methyl esters

Figure 3. Trans esterification and self-metathesis of fatty acid methyl esters 


\section{Results \& Discussion}

Moringa oleifera seed oil with saturated fatty acids, palmitic, stearic, arachidic and behenic acids and monounsaturated fatty acid, oleic acid, (monounsaturated fatty acid, 75\% major) along with minor amounts of palmitoleic, linoleic, linolenic and eicosenoic fatty acids (Table. 1). Self-metathesis at the double bonds of methyl esters of Moringa was carried out to develop a wide range of platform chemicals employing Grubbs' second generation catalyst, $0.3 \mathrm{mM}$. No metathesis reaction was observed when the catalyst concentration was $<0.3 \mathrm{mM}$. However, the concentration of catalyst used was less compared to the reported literature [16]. The metathesized products with 70\% yield were analysed by GC and GC-MS.

A valuable product 9-Octadecene (Ret. Time: $15.87 \mathrm{~min}, 15 \%, \mathrm{GC} ; \mathrm{m} / \mathrm{z}: 252$, GC-MS) was obtained by the reaction between two 9-octadecenoic acid methyl esters (Figure.3, Table. 2 ) which can be dimerised and hydrogenated to give (E)-10,11-dioctyleicosane, a lube-oil range hydrocarbon intermediate [16-17]. This is a motor oil which has potential to decrease the friction between the moving parts [18]. Similar products were also observed in minor amounts in palash with low percentage of unsaturation (65\%) [18]. Other hydrocarbons observed in minor amounts were 6-pentadecene (Ret. Time: $13.07 \mathrm{~min}, 9.3 \% \mathrm{GC} ; \mathrm{m} / \mathrm{z}: 210$, GC-MS) obtained by the reaction between 9-octadecenoic acid methyl ester and 9,12-octadecadienoic acid methyl ester as shown in Figure. 3, Table. 2. 6-Pentadecene is a type of alarm pheromone found in acarid mite [19] and 3-dodecene (Ret. Time: $12.52 \mathrm{~min}, 6.7 \% \mathrm{GC} ; \mathrm{m} / \mathrm{z}$ : 168, GC-MS) observed in major amounts. A bicyclic hydrocarbon, cyclodecacyclotetradecene potential synthetic organic intermediate (Ret. Time: $22.08 \mathrm{~min}, 14 \% \mathrm{GC} ; \mathrm{m} / \mathrm{z} 286$, GC-MS) was obtained by the reaction between two 9,12,15-octadecatrienoic acid methyl esters or two 9,12-octadecadienoic acid methyl esters or 9,12,15-octa decatrienoic acid methyl ester and 9,12-octadecadienoic acid methyl esters which were also observed in most of the self-metathesis reactions of unsaturated fatty acids of non-edible oils [18, 20], Figure.3, Table. 2). A monoester 8,11- eicosadienoic acid methyl ester (Ret.time-14.79 $\mathrm{min}, 9.7 \% \mathrm{GC} ; \mathrm{m} / \mathrm{z} 334$, GC-MS) with unsaturation at unusual positions, a synthetic organic intermediate was obtained by isomerisation of 9,12 octadecenoic acid. A cyclic monoester cyclopropaneoctanoic acid methyl ester (Ret.time-13.09 $\mathrm{min}, 5.1 \% \mathrm{GC}$; $\mathrm{m} / \mathrm{z} \mathrm{200,} \mathrm{GC-MS}$ ) in major amounts were observed compared to metathesis of rubber fatty acid methyl esters [20]. Hence, moringa oil can also be used as an alternative to other edible oils rich in unsaturation, most of the products formed have synthetic importance.

Table 2. Composition of products (wt \%) obtained by self-metathesis of moringa fatty acid methyl esters

\begin{tabular}{|l|l|l|}
\hline \multirow{2}{*}{$\begin{array}{l}\text { Fatty acid methyl } \\
\text { esters }\end{array}$} & \multicolumn{2}{|l|}{ Composition (wt\%) of metathesized products } \\
\cline { 2 - 3 } & Hydrocarbons (wt\%) & Methyl esters (wt\%) \\
\hline \multirow{3}{*}{ Moringa } & 9- Octadecene (15\%) & 8,11- Eicosadienoic acid methyl ester ( 9.7\%) \\
& 6-pentadecene (9.3\%) & Cyclopropaneoctanoic acid methyl ester ( 5.1\%) \\
& $\begin{array}{l}\text { 3-dodecene (6.7\%) } \\
\text { Cyclodecacycltetradecene } \\
(14 \%)\end{array}$ & \\
\hline
\end{tabular}

\section{References}

[1]. J. C. Mol, "Metathesis of unsaturated fatty acid esters and fatty oils," J. Mol. Catal, vol. 90, pp. 185-199, 1994.

[2]. Siegfried Warwel, Falk Bruse, Christoph Demes, Michael Kunz, Mark Rusch gen. Klaas, "Polymers and surfactants on the basis of renewable," Chemosphere, vol. 43, pp. 39-48, 2001.

[3]. U. Biermann, W. Friedt, S. Lang, W. Luhs, et al, "New syntheses with oils and fats as renewable raw materials for the chemical industry," Angew. Chem. Int. Ed. Engl, vol. 39, pp. 2206-2224, 2000.

[4]. Cohen et al. "Methods of refining natural oil feed stocks," US 8957268 B2, 2015.

[5]. J. Behr, Perez Gomes, "The refinement of renewable resources: New important dervatives of fatty acids and glycerol," Eur. J. Lipid Sci. Technol, vol. 31, pp. 112, 2010.

[6]. P. Schwab, M. B. France, J. W. Ziller, R. H. Grubbs, "A series of well defined metathesis catalysts-synthesis of [rucl2(chr)(pr3)2] and its reactions," Angew. Chem. Int. Ed. Engl, vol. 34, pp. 2039-2041, 1995.

[7]. S.L. Scott, "Catalytic transformation of seed oil derivatives via olefin metathesis," HELIA, vol. 30, pp. 133-142, 2007.

[8]. Laleh Jafarpour, Marie-Pierre Heck, Christophe Baylon, Han Man Lee, Charles Mioskowski, P. Steven Nolan, "preparation and activity of recyclable polymer-supported ruthenium olefin metathesis catalysts, " Organometallics, vol. 21, pp. 671-679, 2002.

[9]. E. Verkuijlen C. Boelhouwer "Formation of cyclohexane-1, 4-diene by metathesis of linoleicand linolenic esters," J. Chem. Soc. Chem. Commun, pp. 793-794, 1974.

[10]. K.A. Vikulov, K.A. Shelimov V.B. Kazansky, J.C. Mol, "Deactivation mechanism of photoreduced and cyclopropane-treated molybdena/silica catalysts for olefin metathesis," J. Mol. Catal, vol. 90, pp. 61, 1994.

[11]. Sigl, Marcus, Schneider, Daniel, "Supported catalyst for metathesis of functionalized olefins, especially oleic acid methyl ester," PCT Int. Appl. WO 2008077835 A2 20080703, 2008.

[12]. V. I. Bykov, T. A. Butenko, E. S. Finkel'shtein, and P. T. Henderson, "Synthesis of monoene pheromone components having a double bond in positions 6-11 using cometathesis of cycloolefins with $\alpha$-olefins," Journal of Molecular Catalysis, vol. 90, pp. $111-116,1994$

[13]. J. Tsaknis, V. Spiliotis, S. Lalas, V. Gergis, and V. Dourtoglou, "Quality changes of moringa oleifera, variety mbololo of kenya seed oil during frying”. J. Agric. Food Chem. vol. 47, pp. 4495-4499, 1999.

[14]. Lakshmipriya Gopalakrishnanb, Kruthi Doriyaa, Devarai Santhosh Kumara, "Moringa oleifera: A review on nutritive importance and its medicinal application," Food Science and Human Wellness, vol. 5, pp. 49-56, 2016. 
[15]. Dharmendra Singh, Priya Vrat Arya, Ved Prakash Aggarwal and Radhey Shyam Gupta "Evaluation of antioxidant and hepatoprotective activities of moringa oleifera lam. leaves in carbon tetrachloride-intoxicated rats," Antioxidants, vol. 3, pp. 569591,2014 ,

[16]. M.A.R. Meier, J.O. Metzger, U.S. Schubert, "Plant oil renewable resources as green alternatives in polymer science," Chem. Soc. Rev, vol. 36, pp. 1788-1802, 2007.

[17]. Mol, J.C., "Application of olefin metathesis in oleochemistry: an example of green chemistry," Green Chem. vol. 4, pp. 5-13, 2002.

[18]. Yelchuri Vyshnavi, Rachapudi Badari Narayana Prasad and Mallampalli Sri Lakshmi Karuna, Synthesis of industrially important platform chemicals via olefin metathesis of palash fatty acid methyl esters, European Journal of Chemistry, vol. 5, pp. 532-535, 2014.

[19]. R. Joachim, L.M.S. Johannes, "Mites as matchmakers: semiochemicals from host-associated mites attract both sexes of the parasitoid lariophagus distinguendus," J. Chem. Ecol. vol. 26, pp. 1205-1217, 2000.

[20]. Yelchuri Vyshnavi, R. B. N. Prasad, M. S. L. Karuna, "Metathesis of rubber fatty acid methyl esters: A green approach for generating industrial platform chemicals," IJSR, vol. 3, pp. 209-212, 2014. 